\title{
A COMPLEX AIRY INTEGRAL
}

\section{Dedicated to Professor Tikao Tatuzawa on his 60th birthday}

\section{TOMIO KUBOTA}

The Airy integral is a formula concerning the Fourier transform of a function like $\sin x^{3}$ or $\cos x^{3}$, and is written, for instance in [2], as

$$
\int_{0}^{\infty} \cos \left(t^{3}-x t\right) d t=\frac{1}{3} \pi \sqrt{\frac{1}{3} x}\left[J_{-1 / 3}\left(\frac{2 x \sqrt{x}}{3 \sqrt{3}}\right)+J_{1 / 3}\left(\frac{2 x \sqrt{x}}{3 \sqrt{3}}\right)\right]
$$

for $x \geqq 0$.

In this paper, we shall prove a similar formula

$$
\text { (1) } \int_{C} e\left(z^{3}-3 z w\right) d V(z)=\frac{1}{3} \pi^{2}\left(\sin \frac{\pi}{3}\right)^{-1}|w|\left(\left|J_{-1 / 3}\left(2 \pi w^{3 / 2}\right)\right|^{2}-\left|J_{1 / 3}\left(2 \pi w^{3 / 2}\right)\right|^{2}\right)
$$

containing same Bessel functions and the exponential function $e(z)=$ $\exp (\pi \sqrt{-1}(z+\bar{z}))$, where $d V(z)$ is the usual euclidean measure, and the integral $\int_{c}$ should be interpreted as $\lim _{Y \rightarrow \infty} \int_{|z|<Y}$. This is a byproduct of the results in [1].

The proof of our main result (1) is reduced to an equality between Mellin transforms of certain functions. Let us first treat the purely computational part of the proof. If $z=r \exp (\sqrt{-1} \theta)$ and $w=r^{\prime} \exp \left(\sqrt{-1} \theta^{\prime}\right)$, $\left(r, r^{\prime} \geqq 0, \theta, \theta^{\prime} \in \boldsymbol{R}\right)$, are polar expressions of complex numbers $z$ and $w$, then a general formula on the Bessel function $J_{m}$ says

$$
e(z)=\sum_{m=-\infty}^{\infty} \sqrt{-1}^{m} J_{m}(2 \pi r) \exp (\sqrt{-1} m \theta) .
$$

This implies

$$
\begin{gathered}
e\left(z^{3}\right)=\sum_{m=-\infty}^{\infty} \sqrt{-1}^{m} J_{m}\left(2 \pi r^{3}\right) \exp (\sqrt{-1} 3 m \theta), \\
e(-3 z w)=\sum_{m=-\infty}^{\infty}(-\sqrt{-1})^{m} J_{m}\left(6 \pi r r^{\prime}\right) \exp \left(\sqrt{-1} m\left(\theta+\theta^{\prime}\right)\right),
\end{gathered}
$$

Received June 2, 1975.

This research is supported by NSF Grant GP-43950 (SK-CUCB). 
and consequently

$$
\begin{aligned}
\int_{C} e\left(z^{3}\right) e(-3 z w) d V(z) \\
=\sum_{m=-\infty}^{\infty}\left[2 \pi \int_{0}^{\infty} J_{-m}\left(2 \pi r^{3}\right) J_{3 m}\left(6 \pi r r^{\prime}\right) r d r\right] \exp \left(\sqrt{-1} 3 m \theta^{\prime}\right) \\
=\sum_{m=-\infty}^{\infty}(-1)^{m}\left[2 \pi \int_{0}^{\infty} J_{m}\left(2 \pi r^{3}\right) J_{3 m}\left(6 \pi r r^{\prime}\right) r d r\right] \exp \left(\sqrt{-1} 3 m \theta^{\prime}\right),
\end{aligned}
$$

where $\int_{0}^{\infty}$ is in the sense of $\lim _{Y \rightarrow \infty} \int_{0}^{Y}$.

Denote in general by

$$
M(f, s)=\int_{0}^{\infty} f(y) y^{s} \frac{d y}{y}
$$

the Mellin transform of a function $f$. Then, there are well-known formulas

$$
M\left(J_{m}(\alpha r), s\right)=\alpha^{-s} \frac{2^{s-1} \Gamma(s / 2+m / 2)}{\Gamma(1-s / 2+m / 2)},
$$

$(\alpha>0)$, and

$$
M\left(J_{m}\left(2 \pi r^{3}\right), s\right)=\frac{1}{3}(2 \pi)^{-s / 3} \frac{2^{s / 3-1} \Gamma(s / 6+m / 2)}{\Gamma(1-s / 6+m / 2)} .
$$

On the other hand, $\Gamma$-function satisfies the multiplication formula $\Gamma(s)=$ $(2 \pi)^{-1} 3^{s-1 / 3} \Gamma(s / 3) \Gamma(s / 3+1 / 3) \Gamma(s / 3+2 / 3)$. Using these facts, we can compute the Mellin transform of the function

$$
b_{m}\left(r^{\prime}\right)=2 \pi \int_{0}^{\infty} J_{m}\left(2 \pi r^{3}\right) J_{3 m}\left(6 \pi r r^{\prime}\right) r d r
$$

of $r^{\prime}$ appearing in (2) as follows:

$$
\begin{aligned}
M\left(b_{m}, s\right) & =2 \pi \int_{0}^{\infty} \int_{0}^{\infty} J_{m}\left(2 \pi r^{3}\right) J_{3 m}\left(6 \pi r r^{\prime}\right) r d r r^{\prime s} \frac{d r^{\prime}}{r^{\prime}} \\
& =2 \pi \int_{0}^{\infty} \int_{0}^{\infty} J_{m}\left(2 \pi r^{3}\right) J_{3 m}\left(6 \pi r^{\prime}\right) r d r \frac{r^{\prime s}}{r^{s}} \frac{d r^{\prime}}{r^{\prime}} \\
& =2 \pi \int_{0}^{\infty} J_{m}\left(2 \pi r^{3}\right) r^{2-s} \frac{d r}{r} \int_{0}^{\infty} J_{3 m}\left(6 \pi r^{\prime}\right) r^{\prime s} \frac{d r^{\prime}}{r^{\prime}} \\
& =2 \pi M\left(J_{m}\left(2 \pi r^{3}\right), 2-s\right) M\left(J_{3 m}(6 \pi r), s\right) \\
& =2 \pi \cdot \frac{1}{3}(2 \pi)^{-(2-s) / 3} \frac{2^{(2-s) / 3-1} \Gamma((2-s) / 6+m / 2)}{\Gamma(1-(2-s) / 6+m / 2)}(6 \pi)^{-s} \frac{2^{s-1} \Gamma(s / 2+3 m / 2)}{\Gamma(1-s / 2+3 m / 2)}
\end{aligned}
$$




$$
\begin{aligned}
= & 2 \pi \cdot \frac{1}{3}(2 \pi)^{-(2-s) / 3}(6 \pi)^{-s} 2^{(2-s) / 3-1} 2^{s-1} \frac{\Gamma(1 / 3-s / 6+m / 2)}{\Gamma(2 / 3+s / 6+m / 2)} \cdot \\
& \cdot \frac{3^{s / 2+3 m / 2-1 / 2} \Gamma(s / 6+m / 2) \Gamma(s / 6+m / 2+1 / 3) \Gamma(s / 6+m / 2+2 / 3)}{3^{1-s / 2+3 m / 2-1 / 2} \Gamma(1 / 3-s / 6+m / 2) \Gamma(2 / 3-s / 6+m / 2) \Gamma(1-s / 6+m / 2)} \\
= & \frac{1}{18 \pi} \pi^{-(2 s-4) / 3} \frac{\Gamma(s / 6+m / 2) \Gamma(s / 6+m / 2+1 / 3)}{\Gamma(2 / 3-s / 6+m / 2) \Gamma(1-s / 6+m / 2)} .
\end{aligned}
$$

Comparing this result with Proposition 1 of [1], one sees by Theorem 1 of [1] that the coefficients of $\exp \left(\sqrt{-1} m \theta^{\prime}\right)$ in the Fourier series expansion with respect to $\theta^{\prime},\left(w=r^{\prime} \exp \left(\sqrt{-1} \theta^{\prime}\right)\right)$, of the both hand sides of (1) have a common Mellin transform for $m \geqq 0$. Since, however, $e(z)=e(\bar{z})$ implies that the left hand side of (1) is invariant by $w \rightarrow \bar{w}$, the same situation holds for $m<0$, too.

To complete the proof, we now need only a few supplements to the above computation. Introducing a parameter $\rho$, let us consider the integral

$$
2 \pi \int_{0}^{\infty} \int_{0}^{\infty} J_{m}\left(2 \pi r^{3}\right) J_{3 m}\left(6 \pi r r^{\prime}\right) r^{\rho} d r r^{\prime s} \frac{d r^{\prime}}{r^{\prime}}
$$

Then, under the condition, for instance, $0<\operatorname{Re} s<\varepsilon$ and $-\varepsilon<\operatorname{Re} \rho<0$ with a small positive $\varepsilon, \operatorname{Re}(1+\rho-s)$ is slightly smaller than 1. (As a matter of fact, it will be enough that $\operatorname{Re}(1+\rho-s)$ is close to 1.) Therefore, the same computation as above shows that (3) is equal to the absolutely convergent integral

$$
2 \pi \int_{0}^{\infty} J_{m}\left(2 \pi r^{3}\right) r^{1+\rho-s} \frac{d r}{r} \int_{0}^{\infty} J_{3 m}\left(6 \pi r^{\prime}\right) r^{\prime s} \frac{d r^{\prime}}{r^{\prime}}
$$

which can be expressed as

$$
\begin{aligned}
& 2 \pi M\left(J_{m}\left(2 \pi r^{3}\right), 1+\rho-s\right) M\left(J_{3 m}(6 \pi r), s\right) \\
& \quad=2 \pi \cdot \frac{1}{3}(2 \pi)^{-(1+\rho-s) / 3} \frac{2^{(1+\rho-s) / 3} \Gamma((1+\rho-s) / 6+m / 2)}{\Gamma(1-(1+\rho-s) / 6+m / 2)}(6 \pi)^{-s} \frac{2^{s-1} \Gamma(s / 2+3 m / 2)}{\Gamma(1-s / 2+3 m / 2)}
\end{aligned}
$$

in terms of $\Gamma$-functions, and has the inverse Mellin transform

$$
2 \pi \int_{0}^{\infty} J_{m}\left(2 \pi r^{3}\right) J_{3 m}\left(6 \pi r r^{\prime}\right) r^{\rho} d r
$$

in the region determined by $0<\operatorname{Re} s<1$, say. Considering the analytic continuation to $\rho=1$, we see now that $b_{m}$ is actually the inverse Mellin 
transform in the region $0<\operatorname{Re} s<\varepsilon$ of $M\left(b_{m}, s\right)$, which has been computed formally.

Remark. A simpler integral similar to (1) is

$$
\int_{C} e\left(z^{2}\right) e(z w) d V(z)=\frac{1}{2} e\left(-\frac{1}{4} w^{2}\right)
$$

\section{REFERENCES}

[1] T. Kubota, On a generalized Fourier transformation, to appear in J. Fac. Sci. Univ. Tokyo.

[2] G. N. Watson, A treatise on the theory of Bessel functions, Cambridge University Press, 1966.

Department of Mathematics, Nagoya University

Department of Mathematics, University of Maryland 